\section{Measurement and definition for smoking cessation intervention research: the Smoke-Free Families experience}

Cathy L Melvin, Pattie Tucker, and the Smoke-Free Families Common Evaluation Measures for Pregnancy and Smoking Cessation Projects Working Group*
Cecil G Sheps Center for Health Services Research, University of North Carolina at Chapel Hill, Chapel Hill, North Carolina, USA

C L Melvin

Division of

Reproductive Health, National Center for Chronic Disease

Prevention and Health Promotion, Centers for Disease Control and Prevention,

Atlanta, Georgia, USA

P Tucker

^American Cancer Society Elaine Arkin; Centers for Disease Control and

Prevention, Division of Reproductive Health Juliette Kendrick, Ken

Kolodner, Cathy Melvin, Gary Stuart, Pattie

Tucker; Robert Wood

Johnson Foundation

C Tracy Orleans;

Smoke-Free Families

National Program

Office (University of

Alabama at

Birmingham) $\mathrm{H}$

Pennington Whiteside, Jr, Richard Windsor;

Smoke-Free Families

National Advisory

Committee Dianne C

Barker, Ellen Gritz;

Smoke-Free Families

Grantees Patricia

Dolan-Mullen, Laura

Solomon; University of

Maryland-Baltimore

County Carlo C

DiClemente

Correspondence to:

Cathy L Melvin, PhD, MPH,

Cecil G Sheps Center for

Health Services Research,

University of North

Cniversity of North

Airport Road, Chapel Hill

Airport Road, Chapel Hill,

cathy_melvin@unc.edu

\begin{abstract}
The measures, definitions, and processes used in the Smoke-Free Families clinical trials to assure consistent measurement and reporting of various aspects of the trials are described. Definitions of current smokers at different points in the pregnancy, levels of addiction, biological verification, cessation, stages of change, and intervention approaches are presented along with the rationale underlying their adoption and development.

(Tobacco Control 2000;9(Suppl III):iii87-iii90)
\end{abstract}

Keywords: smoking cessation; pregnancy; clinical trials; addiction

The purpose of the Smoke Free Families (SFF) project is to identify innovative best practices for achieving smoking cessation before, during, and after pregnancy. Eleven clinical trials were undertaken with a wide variety of promising interventions. To assure consistency across all of the trials and to facilitate meta-analysis of the results from these trials, the Smoke Free Families National Program Office (NPO) and the Centers for Disease Control and Prevention (CDC) defined common measures and processes to be applied across all projects. This paper presents these measures and processes.

\section{Methods}

The literature on smoking cessation during pregnancy was reviewed to identify measures used for assessment of smoking status in the prenatal, preconceptional, and postpartum periods, biochemical verification of smoking status, determination of cessation, definition of the stages of pregnancy quitting, and documentation of the implementation of the intervention, including provider time. A working group met in September of 1995 to review the material and to achieve consensus on the most useful measures. The group was established by the CDC and SFF with membership from the American Cancer Society, the CDC, the Robert Wood Johnson Foundation, SFF NPO, SFF National Advisory Committee (NAC), SFF grantees, and invited experts. Comments from all SFF grantees were solicited and modifications to the measures were made as appropriate. Guid- ance for all SFF grantees was then distributed in advance of trial initiation.

\section{Assessment of smoking status}

Questions were chosen, adapted or developed to assess smoking status at two different points in time: (1) at the time of the first prenatal visit (prenatal screening), including questions about smoking before pregnancy (preconceptional); and (2) at some specified time following the end of pregnancy (postpartum screening). The questions listed in box 1 were used for prenatal assessments and, in box 2, for postpartum assessments.

PRENATAL SCREENING

The purpose of the prenatal screening questions is to identify pregnant smokers at the time they enter prenatal care. Accurate assessment of smoking status at the first prenatal visit identifies smokers for participation in the trial and establishes a baseline measure of smoking status for each enrolled participant and of smoking prevalence for the study population. Some studies also used these questions in subsequent assessments of smoking status, as specified by the trial protocol. All trials, at a minimum, were required to ask these questions at the first prenatal visit. A second assessment was required at or near the end of pregnancy to establish changes in smoking status during the pregnancy. The timing of the postpartum assessment varied with the study design. The suggested order of these questions was based on current knowledge of approaches to minimise non-disclosure of smoking status. ${ }^{1}$

MEASURES OF CURRENT SMOKING STATUS

At least two, and as many as four, questions about current smoking status were asked of the pregnant woman during her first prenatal visit (box 1).

Responses to questions 1, 2, and 3 allowed each woman to be categorised as a "smoker" or a "non-smoker". Question 1 is designed to minimise non-disclosure of smoking status and to provide investigators more specific information about current smoking behaviour. Many pregnant women are reluctant to disclose their smoking status at the first prenatal visit. Deception rates as high as $23 \%$ among Medicaid insured $^{12}$ and $14 \%$ among privately 
Box 1. Recommended questions for prenatal screening

1. Current smoking status

Which statement best describes you now?

a. I smoke regularly now - about the same amount as BEFORE I found out I was pregnant.

b. I smoke regularly now, but more than BEFORE I found out I was pregnant.

c. I smoke some now, but I have cut down SINCE I got pregnant.

d. I stopped smoking AFTER I found out I was pregnant, and I am not smoking now.

e. I stopped smoking BEFORE I found out I was pregnant, and I am not smoking now.

f. I have NEVER smoked more than 100 cigarettes.

2. Current smoking status

Have you had a cigarette, even a puff, within the last 30 days?

a. Yes b. No

3. Current smoking status

Have you had a cigarette, even a puff, within the last 7 days?

a. Yes b. No

4. Current smoking quantity (optional)

During the past 7 days, how many cigarettes or packs of cigarettes did you smoke on an average day? (A pack has 20 cigarettes) _cigarettes or _packs ( ) Less than 1 cig/day ( ) Didn't smoke ( ) Don't know

5. Addiction measure (optional)

How soon after you wake up do you usually smoke your first cigarette?

_ Immediately _ minutes (number) __Hours (number)

_No usual time/time varies __Don't know

6. Baseline biochemical measurements (optional)

7. Preconceptional smoking status

In the past 3 months before you got pregnant, how many cigarettes or packs of cigarettes did you smoke on an average day? (A pack has 20 cigarettes)

cigarettes or _ packs ( ) less than $1 \mathrm{cig} /$ day ( ) Didn't smoke ( ) Don't know

Box 2. Recommended supplementary questions for postpartum screening

8. Current smoking status

Did you smoke cigarettes at all during the 12 months before your delivery?

( )Yes-Go to next question ( )No

9. Smoking status during pregnancy

In the 3 months after you got pregnant, how many cigarettes or packs of cigarettes did you smoke on an average day? (A pack has 20 cigarettes)

cigarettes or _packs ( ) Less than $1 \mathrm{cig} /$ day ( ) Didn't smoke

( ) Don't know
30 days, she was then asked question 3 to determine if she had smoked, even a puff, within the previous seven days. This question is frequently used to determine if a person is currently smoking. " The answer of "yes" to question 3 and of "a", " $b$ " or "c" to question 1 resulted in the classification of the pregnant woman as a "smoker". At this point, she was given an opportunity to enroll in the smoking cessation program. If she chose response " $\mathrm{d}$ ", "e" or "f" to question 1 and answered "no" to question 2 , she was considered a "nonsmoker" by self report.

Questions 4 and 5 were optional questions to be asked of pregnant smokers. They were used to measure the level of addiction based on the quantity of cigarettes smoked during a typical day and the timing of the first cigarette of the day. ${ }^{4}$ Question 5 may need to be adjusted for special populations such as teens who may not be in situations that let them behave as they would in unsupervised situations. For example, teens may postpone their first cigarette of the day in instances where they live at home and want to avoid smoking in front of their parents.

\section{BIOCHEMICAL VERIFICATION OF SELF REPORTS} (OPTIONAL)

If possible, biochemical verification of self reported smoking status was done at the time the other questions were asked (box 1, item 6 ). Baseline biochemical verification is essential in studies that have a major intervention goal of significantly reducing cigarette consumption by pregnant smokers. Investigators were asked to report exactly when, in relation to the asking of the prenatal screening questions, they informed the pregnant woman that biochemical verification of her smoking status was being undertaken.

Measures and tests that could be used by investigators to confirm self reports include urinary or saliva cotinine and expired carbon monoxide. The cut off level for determining active, rather than passive, exposure to cigarette smoke for urinary cotinine is $>80 \mathrm{ng} /$ $\mathrm{ml}$, for saliva cotinine is $>30 \mathrm{ng} / \mathrm{ml}$, and for expired carbon monoxide is $>9$ parts per million. ${ }^{56}$ Creatinine adjustment of urinary cotinine is not necessary when defining smoking status for a population of pregnant women, since adjustments do not affect classification of women as smokers or quitters. However, in studies of reduction, or in dealing with individual women, creatinine adjustments may be necessary, especially for women who smoke very little or may only have passive exposure. Baseline cotinine concentrations have been shown to vary by race, with higher cotinine concentrations observed in black rather than in white smokers. These differences are significant and are not explained by reporting bias or nicotine intake difference. However, these differences do not affect choice of cut off values for determining active smoking. ${ }^{7}$

MEASURES OF PRECONCEPTIONAL SMOKING STATUS (OPTIONAL)

The preconceptional questions were used to obtain information about the pregnant 
Box 3. Staging pregnancy quitting

10. Have you smoked any cigarettes in the past 7 days, even a puff? Yes-Go to next question $\mathrm{No}=$ Action

11. If yes, are you seriously thinking about quitting completely during this pregnancy?

Yes-Go to next question $\mathrm{No}=$ Precontemplation

12. If yes, are you planning to quit completely in the next 30 days? Yes $=$ Preparation $\quad$ No $=$ Contemplation

woman's smoking status and level of addiction in the three months before conception. In most cases these questions are asked retrospectively of women who are just beginning their prenatal care.

Two questions were suggested but not required. The first, question 7 , was taken from the pregnancy risk assessment monitoring system (PRAMS), an ongoing, state specific, population based surveillance system designed to identify and monitor selected maternal behaviours and experiences before, during, and after pregnancy. ${ }^{8}$ Responses to this question allow researchers to estimate the prevalence of self reported smoking among the study population before pregnancy and to categorise smokers on the basis of the quantity smoked. These measures can then be used with other indicators to identify spontaneous quitters and to observe self reported reductions in the numbers of cigarettes smoked.

It was also suggested that question 5 be modified to assess level of addiction before pregnancy rather than level of addiction at the current time as specified in the questions for the prenatal assessment of smoking status.

POSTPARTUM SCREENING

The postpartum screening questions are used to identify current smokers during the postpartum period and to assess smoking behaviours before and during pregnancy. As presented here, they are intended for use in studies where enrollment occurs only at the postpartum visit.

Prenatal screening questions 3,4 , and 5 (box 1) are suggested for postpartum assessments along with questions 8 and 9 (box 2). Questions 3, 4, and 5 are used to determine current smoking status, quantity smoked, and level of addiction at the postpartum visit. Women were classified as smokers if they indicated smoking a cigarette, even a puff, within the last seven days. Question 8 provides a self report of smoking during the pregnancy, and question 9 of quantity smoked during the first three months of pregnancy.

Biochemical verification of self reported smoking status is also suggested for postpartum assessments.

DEFINITION OF CESSATION

The measure of success for the SFF trials was biochemically verified cessation. The process and questions described below were used to identify pregnant smokers who quit smoking cigarettes.

- Quit: A pregnant smoker could be classified as a quitter only if the following conditions were met: (1)The pregnant smoker must report cessation by answering "no" to the question "Have you had a cigarette, even a puff, in the last 7 days?" (Question 3, Box 1 ); and (2) there must be a biochemical verification of her smoking status indicating that she has not smoked within the period covered by the biochemical marker in use.

- Timing of quit (optional): The following question was suggested to ascertain the timing of cessation, especially if the study focused on an outcome in which documentation of total smoking exposure during pregnancy could be important:

When did you smoke your last cigarette?

______ (month/day/year)

- Relapse (optional): This question was suggested to determine if the pregnant woman relapsed at all since her most recent cessation attempt. It is especially important in studies using stage tailored counselling:

Have you smoked at all, even a puff, since you last quit? ( ) Yes ( ) No

- Quit attempts before and after pregnancy (optional): The following questions were developed by the working group to assess the cycling of quitting and its relevance to the pregnant woman's ability to quit:

(a) How many times had you quit smoking for more than 24 hours before you found out about this pregnancy?
None Times
Don't know

(b) How many times have you quit smoking for more than 24 hours since you found out about this pregnancy?

None Times

Don't know

\section{STAGING PREGNANCY QUITTING}

For those trials using stage tailored behavioural interventions, standard questions were used to determine the stage of readiness for each pregnant smoker. Smokers were classified as being in one of four stages: precontemplation, contemplation, preparation or action, based on their responses to the questions in box 3 . The stage assessments occurred at enrollment and at postpartum follow up as well as at selected intervals between these two points. Interim measurements may not have been necessary or helpful for some intervention designs.

DOCUMENTATION OF INTERVENTION

Documentation of the various components of the intervention and of how it is implemented is critically important when evaluating program performance and/or considering replication of the program. To assure that programs could be adequately described and that program fidelity had been achieved in the trial, the following information was required and collected for each intervention:

- A thorough description of program components, methods and training

- Documentation of the type, content and distribution schedule of self help materials provided to smokers

- Descriptions of the pregnant smokers' use and ratings of self help materials provided to them 
- Documentation of the pregnant smoker's compliance with intervention proto$\mathrm{col}(\mathrm{s})$, including their use of various methods and materials

- Documentation of the data management process for assuring accuracy in data collection

DOCUMENTATION OF PROVIDER TIME SPENT IMPLEMENTING INTERVENTIONS

Given the significant time constraints faced by providers, it is important to document the time required to deliver interventions. A set of questions was developed by the working group to document: (1) how much of each type of provider's time it actually took to implement a particular prenatal smoking cessation intervention; and (2) the sequencing of the intervention in relation to other activities occurring during the visit. Answers to these questions are important for purposes of quality assurance, clinic efficiency, and cost estimation as well as for future replication efforts.

Documentation of the following components was suggested but not required:

- Description of the particular component of the intervention being delivered by each provider

- Description of the content and method used in implementing each intervention component

- Documentation of the amount of time actually spent with the pregnant woman by each provider and the content of each interaction

- Description of the type of provider (for example, level of experience, training) implementing each component of the intervention

- Salary range for each type of provider

\section{Conclusion}

In the mid 1980s, a review of the literature on smoking cessation treatments for pregnant women resulted in a call for utilising scientific criteria for rigorous, valid research into ways to educate the pregnant smoker. ${ }^{9}$ When the SFF trials began in 1995, only a few approaches had been rigorously evaluated and did not, either collectively or individually, point the way for evidence based practice for smoking cessation treatments for pregnant women. The SFF trials were designed, in part, to address this need for rigorous studies with clear implications for practice. They were also designed to be consistent in their definitions, measures, and processes. Definitions of current smokers at different points in the pregnancy and following it, levels of addiction, biological verification, cessation, stages of change, and intervention approaches created a template for the exchange of information and participation in subsequent meta-analyses. Rigorous and consistently applied processes and measures allowed the SFF investigators to meet the standards required for developing the most defensible evidence of intervention efficacy and for increasing the likelihood that findings from these trials would be useful in developing effective treatments for pregnant smokers.

While this information should be helpful to researchers in understanding and interpreting SFF findings, it may also serve as a useful construct for future work in this area. New approaches to some of the assessments used here are currently being explored. For example, new approaches to asking women about their smoking status were recently reported. ${ }^{10}$ As refinements and enhancements are made, they should be systematically incorporated into future efforts to evaluate the effectiveness of cessation treatments for pregnant women.

1 Windsor RA. Healthcare delivery issues and systems. Presentation at a consensus workshop on smoking cessation in
pregnancy. Health Resources and Services Administrapregnancy. Health Resources and Services
tion, Rockville, Maryland, 9-10 April 1998 .

2 Windsor RA, Woodby LA, Miller T, et al. Effectiveness of AHCPR practice recommendations and patient education methods for pregnant smokers in Medicaid maternity care. Am $\mathcal{F}$ Obstet Gynecol In press.

3 Mullen PD, Carbonari JP, Tabak ER, et al. Improving the disclosure of smoking by pregnant women. Am $\mathcal{F}$ Obstet Gynecol 1991;165:409-13.

4 Fagerstrom KO, Schneider NG. Measuring nicotine dependence: a review of the Fagerstrom tolerance questionnaire. F Behav Med 1989;12:159-82.

5 Kendrick JS, Zahniser SC, Miller N, et al. Integrating smoking cessation into routine public prenatal care: the smoking cessation in pregnancy project. Am $\mathcal{F}$ Public Health 1995;85:217-22.

6 Windsor RA, Orleans TC. Guidelines and methodological standards for smoking cessation interventions research among pregnant women: improving the science and art. Health Educ $Q$ 1986;13:131-61.

7 Wagenknecht LE, Cutter GR, Haley NJ, et al. Racial differences in serum cotinine levels among smokers in the coronary artery risk development in (young) adults study. $\mathrm{Am}$ f Public Health 1990;80:1053-6.

8 Centers for Disease Control and Prevention. PRAMS surveillance report. Atlanta, Georgia: Division of Reproductive Health, National Center for Chronic Disease Prevention and Health Promotion, Centers for Disease Control and Prevention, 1999

9 Windsor RA, Orleans CT. Guidelines and methodological standards for smoking cessation interventions research among pregnant women: Improving the science and art. Health Educ Q 1986;13:131-61.

10 Kharrazi M, Epstein D, Hopkins B, et al. Evaluation of four maternal smoking questions. Public Health Rep 1999; 114:60-70. 